\title{
A finite element method for fluid-structure interaction with surface waves using a finite increment calculus formulation
}

E. Oñate

J. Garcia 


\title{
A FINITE ELEMENT METHOD FOR FLUID-STRUCTURE INTERACTION WITH SURFACE WAVES USING A FINITE INCREMENT CALCULUS FORMULATION
}

\author{
E. Oñate and J. García \\ International Centre for Numerical Methods in Engineering \\ Universidad Politécnica de Cataluña \\ Gran Capitán s/n, 08034 Barcelona, Spain \\ e-mail: onate@cimne.upc.es
}

\section{Abstract}

A stabilized semi-implicit fractional step finite element method for solving coupled

fluid-structure interaction problems involving free surface waves is presented. The stabilized governing equations for the viscous fluid and the free surface are derived at a differential level via a finite increment calculus procedure. A mesh updating technique based on solving a fictitious elastic problem on the moving mesh is described. Examples of the efficiency of the stabilized semi-implicit algorithm for the an alysis of fluid-structure interaction presented.

\section{Introduction}
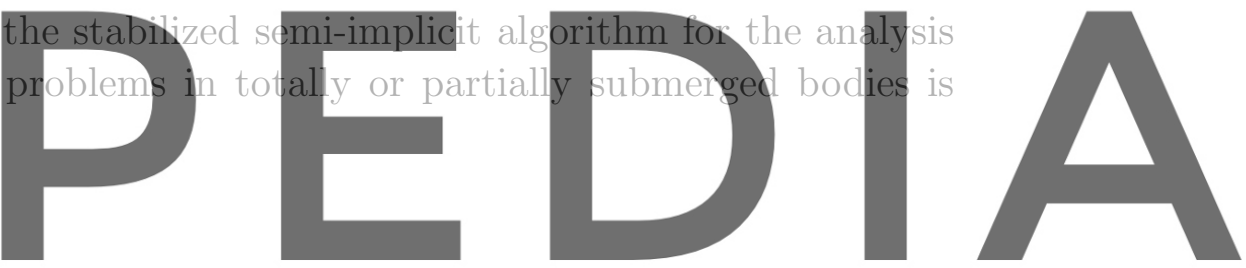

Register for free at https//www.scipedia.com to download the version without the watermark

Accurate prediction of the fluid-structure interaction effects for a totally or partially submerged body in a flowing liquid including a free surface is a problem of great relevance in civil and offshore engineering and naval architecture among many other fields.

The difficulties in accurately solving the coupled fluid-structure interaction problem in this case are mainly due to the following reasons:

1. The difficulty of solving numerically the incompressible fluid dynamic equations which typically include intrinsic non linearities except for the simplest and limited potential flow model.

2. The obstacles in solving the constraint equation stating that at the free surface boundary the fluid particles remain on that surface which position is in turn unknown.

3. The difficulties in solving the problem of motion of the submerged body due to the interaction forces while minimizing the distorsion of the finite elements discretizing the fluid domain, thus reducing the need of remeshing. 
This paper extends recent work of the authors [1-3] to derive a stabilized finite element method which allows to overcome above three obstacles. The starting point are the modified governing differential equations for the incompressible viscous flow and the free surface condition incorporating the necessary stabilization terms via a finite increment calculus (FIC) procedure developed by the authors [4-9]. The FIC approach has been successfully applied to the finite element and meshless solution of a range of advective-diffusive transport and fluid flow problems [1-12].

The stabilized governing equations are written in an arbitrary lagrangian-eulerian (ALE) form to account for the effect of relative movement between the mesh and the fluid points. These equations are solved in space-time using a semi-implicit fractional step approach and the finite element method (FEM). Free surface wave boundary effects are accounted in the flow solution either by moving the free surface nodes in a lagrangean manner, or else for via the introduction of a prescribed pressure at the free surface computed from the wave height.

The movement of a fully or partially submerged body within the fluid due to the interaction forces is treated by solving a structural dynamic problem using the fluid forces as input loads. A method to update the mesh for the fluid domain following the movement of the submerged body which minimizes element distorsion is presented. The mesh update procedure is based on the finite element solution of a linar elastic problem on the mesh anain whore fictitions elastic propentios tue a linear elastic problem on the mesh a assigned so that elements sufferng higher movements are stiffer [13]. The content of the paper is structured as follows. First details of the stabilized
form of the governing equations for a viscous flow and the free surface ysing a finite

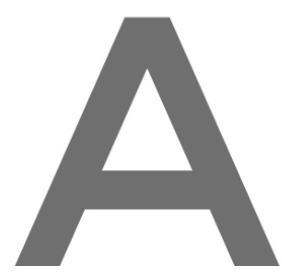
increment calculus procedure are given. The semi-implicit fractional step approach
using the FEM is then described. Details of the computation of the stabilization for free at https/dwww.scipedia.com to download the version without the watermark some examples of free surface wave problems and coupled fluid-interaction problems are given.

\section{Finite increment calculus formulation of fluid- flow and free surface equations}

Finite element solution of the incompressible Navier-Stokes equations with the classical Galerkin method may suffer from numerical instabilities from two main sources. The first is due to the advective-diffusive character of the equations which induces oscillations for high values of the velocity. The second source has to do with the mixed character of the equations which limits the choice of finite element interpolations for the velocity and pressure fields.

Solutions of these two problems have been extensively sought in the last years. Compatible velocity-pressure interpolations satisfying the inf-sup condition emanating from the second problem above mentioned have been used $[14,15,40]$. In addition, the advective operator has been modified to include some "upwinding" 
effects [16-23]. Recent procedures based on Galerkin Least Square [24,25], Characteristic Galerkin [26,27], Variational Multiscale [28-30] and Residual Free Bubbles [31-33] techniques allow equal order interpolation for velocity and pressure by introducing a Laplacian of pressure term in the mass balance equation, while preserving the upwinding stabilization of the momentum equations. Most of these methods lack enough stability in the presence of sharp layers transversal to the velocity. This defficiency is usually corrected by adding new "shock capturing" stabilization terms to the already stabilized equations [35-37]. The computation of the stabilization parameters in all these methods is based in "ad hoc" generalizations of the parameters for the 1D linear advective-diffusive-reactive problem [38,39].

This paper presents a different point view for deriving stabilized finite element methods for incompressible flow problems with a free surface. The starting point are the stabilized form of the governing differential equations derived via a finite increment calculus (FIC) procedure. This technique first presented in [4,5] is based on writting the different balance equations over a domain of finite size and retaining higher order terms. These terms incorporate the ingredients for the necessary stabilization of any transient and steady state numerical solution already at the differential equations level. Application of the standard Galerkin formulation to the consistently modified differential equations for the fluid flow problem leads to a stabilized system of discretized equations which overcomes the two problems above mentioned (i.c. the advective type instab and pressure fields). leads to a new stabilizer solved numerically by standard Galerkin FEN .
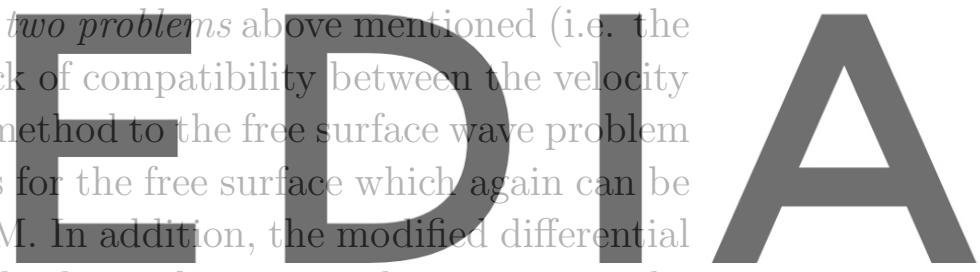

equations can be used to derive a numerical scheme for iteratively computing the

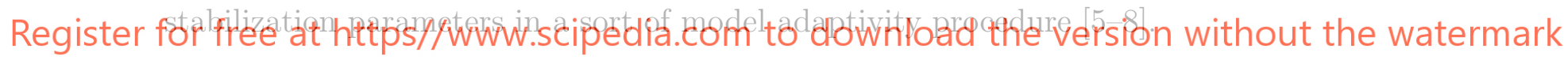

For the sake of preciseness the basic ideas of the FIC method are given next.

\subsection{Basic concept of the finite increment calculus (FIC) method}

Let us consider a sourceless transient problem over a one dimensional domain $A B$ of length $L$ (Figure 1). The balance of flux $q$ over a domain of finite size belonging to $L$ can be written as

$$
q_{A}-q_{B}=0
$$

where $A$ and $B$ are the end points of the finite size domain of length $h$. As usual $q_{A}$ and $q_{B}$ represent the values of the flux $q$ at points $A$ and $B$, respectively.

For instance, in an 1D advective-diffusive problem the flux $q=-c u \phi+k \frac{d \phi}{d x}$, where $\phi$ is the transported variable (i.e. the temperature in a thermal problem), $u$ is the advective velocity and $c$ and $k$ are the advective and diffusive material parameters, respectively. 
The flux $q_{A}$ can be expressed in terms of the values at point $B$ by the following Taylor series expansion

$$
q_{A}=q_{B}-\left.h \frac{\partial q}{\partial x}\right|_{B}+\left.\frac{h^{2}}{2} \frac{d^{2} q}{d x^{2}}\right|_{B}+O h^{3}
$$

Substituting (2) into (1) gives after simplification and neglecting cubic terms in $h$

$$
\frac{d q}{d x}-\underline{\frac{h}{2} \frac{d q}{d x}}=0
$$

where all terms are evaluated at the arbitrary point $B$.

Eq. (3) is the finite increment form of the balance equation over the domain $A B$. The underlined term in eq.(3) introduces the necessary stabilization for the discrete solution of eq.(3) using any numerical technique. Distance $h$ is the characteristic length of the discrete problem and its value depends on the parameters of discretization method chosen (such as the grid size $[1,2]$ ). Note that for $h \rightarrow 0$ the standard infinitesimal form of the balance equation $\left(\frac{d q}{d x}=0\right)$ is recovered.

Above process can be extended to derive the stabilized balance differencial equations for any problem in mechanics as
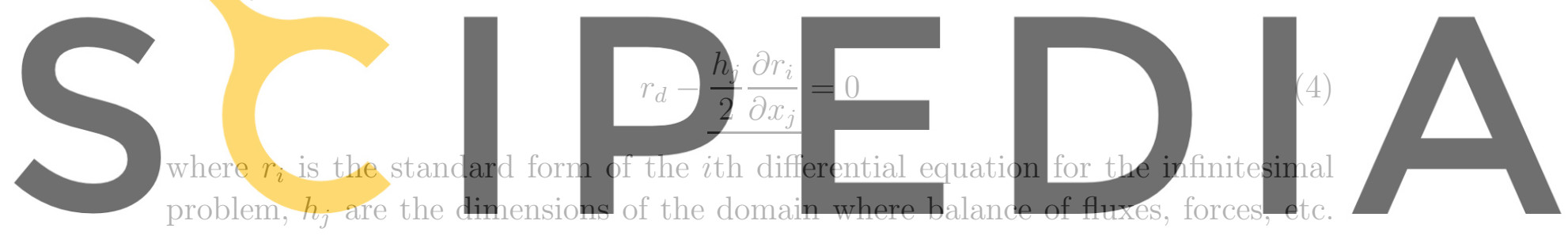

is enforced, and $j=1,2,3$ for 3D problems. Details of the derivation of eq.(4) for

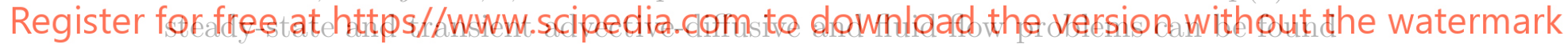

in [4]. Applications of the FIC approach to the Galerkin finite element solution of these problems are reported in $[4-8]$.

The underlined stabilization terms in eqs.(3) and (4) are a consequence of accepting that the infinitesimal form of the balance equations is an unreachable limit within the framework of a discrete numerical solution. Indeed eqs.(3) or (4) are not longer valid for obtaining an analytical solution following traditional integration methods from calculus theory. The meaning of the new stabilized equations makes only sense in the context of a discrete numerical method yielding approximate values of the solution at a finite set of points within the analysis domain. Convergence to the exact analytical value at the points will occur only for the limit case of zero grid size (except for some simple 1D problems $[1,14]$ ) which also implies naturally a zero value of the characteristic length parameters.

The FIC formulation presented below for incompressible flows with a free surface can be considered an extension of that recently developed in [8] for finite element analysis of incompressible Navier-Stokes flows. A new formulation of the stabilized governing differential equations via the FIC method is here presented which holds for the viscous (Stokes) and zero viscosity (Euler) cases. The stabilized fluid flow 
equations are completed by the FIC form of the free surface wave equation following the ideas first presented in [12]. The set of stabilized governing equations is first discretized in time and then solved in space using a Galerkin finite element method. A semi-implicit fractional step procedure is used for the momentum and mass balance equations allowing for a equal order linear interpolations of the velocity and pressure variables over tetrahedral elements.

\subsection{FIC formulation of viscous flow and free surface equa- tions}

We consider the motion around a body of a viscous incompressible fluid including a free surface.

The stabilized FIC form of the governing differential equations for the three dimensional (3D) problem can be written in arbitrary lagrangian-eulerian (ALE) form as

$[8,12]$

\section{Momentum}
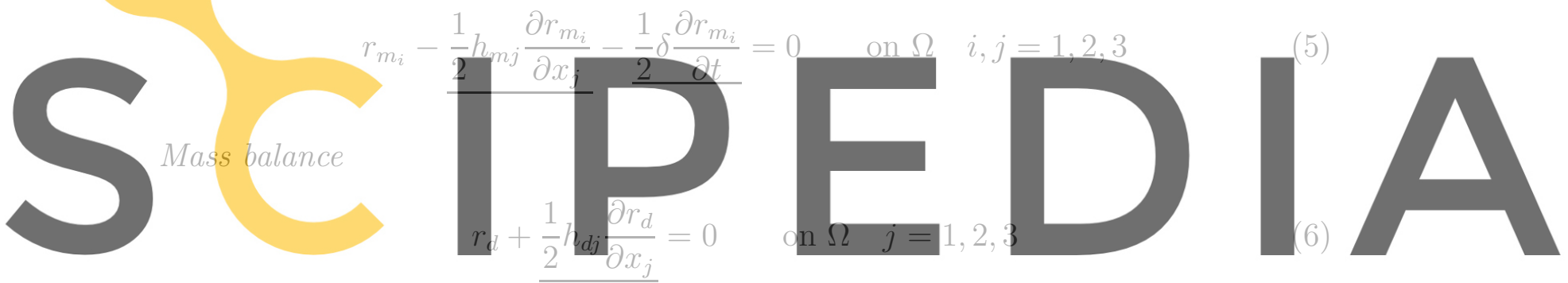

Register forefreefatehttps//www.scipedia.com to download the version without the watermark

$$
r_{\beta}-\underline{\frac{1}{2} h_{\beta_{j}} \frac{\partial r_{\beta}}{\partial x_{j}}}-\underline{\frac{1}{2}} \gamma \frac{\partial r_{\beta}}{\partial t}=0 \quad \text { on } \Gamma_{\beta} \quad j=1,2
$$

where

$$
\begin{aligned}
r_{m_{i}} & =\rho\left[\frac{\partial u_{i}}{\partial t}+\frac{\partial}{\partial x_{j}}\left(v_{i} u_{j}\right)\right]+\frac{\partial p}{\partial x_{i}}-\frac{\partial \tau_{i j}}{\partial x_{j}}-b_{i} \\
r_{d} & =\frac{\partial u_{i}}{\partial x_{i}} \quad i=1,2,3 \\
r_{\beta} & =\frac{\partial \beta}{\partial t}+v_{i} \frac{\partial \beta}{\partial x_{i}}-v_{3} \quad i=1,2 \\
\text { and } & \\
v_{i} & =u_{i}-u_{i}^{m}
\end{aligned}
$$

In above $u_{i}$ is the velocity along the $i$-th global reference axis, $u_{i}^{m}$ is the velocity of the mesh nodes and $v_{i}$ is the relative velocity between the moving mesh and the fluid point $i, \rho$ is the (constant) density of the fluid, $p$ is the pressure, $\beta$ is the wave 
elevation, $b_{i}$ are the body forces acting in the fluid and $\tau_{i j}$ are the viscous stresses related to the viscosity $\mu$ by the standard expression

$$
\tau_{i j}=\mu\left(\frac{\partial u_{i}}{\partial x_{j}}+\frac{\partial u_{j}}{\partial x_{i}}-\delta_{i j} \frac{2}{3} \frac{\partial u_{k}}{\partial x_{k}}\right)
$$

The boundary conditions for the stabilized problem are written as

$$
\begin{aligned}
n_{j} \tau_{i j}+t_{i}+\frac{1}{2} h_{m j} n_{j} r_{m_{i}} & =0 \quad \text { on } \Gamma_{t} \\
u_{j}-u_{j}^{p}=0 & \text { on } \Gamma_{u}
\end{aligned}
$$

where $n_{j}$ are the components of the unit normal vector to the boundary and $t_{i}$ and $u_{j}^{p}$ are prescribed tractions and displacements on the boundaries $\Gamma_{t}$ and $\Gamma_{u}$, respectively.

The underlined terms in eqs.(5)-(7) introduce the necessary stabilization for the approximated numerical solution.

The characteristic length distances $h_{m j}, h_{d j}$ and $h_{\beta_{j}}$ represent the dimensions of the finite domain where balance of momentum, mass and transport of fluid particles is enforced. The signs before the stabilization terms in eqs.(5)-(7) and (13) ensure a positive value of the characteristic length distances. The parameters $\delta$ and $\gamma$ in eqs.(5) and (7) have dimensions of time. Detals of the derivation of eqs. (5)-(7) can be found in $[1,7,8,12]$.
Eqs. (5-14) are the starting point for deriving a variety of stabilized numerical nethods for solving the incompressible Navier-Stokes equations with a free surface. It

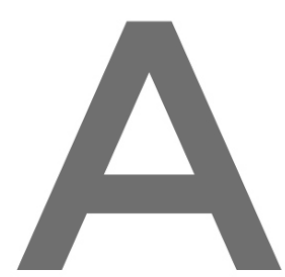

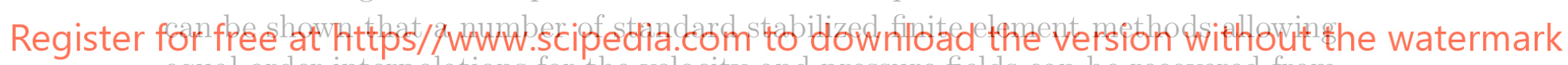
equal order interpolations for the velocity and pressure fields can be recovered from

the modified form of the momentum and mass balance equations given above [8].

\section{Remark 1}

In reference [8] a modified version of the Dirichlet condition (14) is used including an additional stabilization term. This term is not strictily necessary for the subsequent derivation and will be neglected here.

\subsection{Alternative form of the mass balance equation}

Taking the first derivative of eq.(12) gives (assuming the viscosity $\mu$ to be constant)

$$
\frac{\partial \tau_{i j}}{\partial x_{j}}=\mu \Delta u_{i}+\frac{\mu}{3} \frac{\partial r_{d}}{\partial x_{i}}
$$

where $\Delta=\frac{\partial^{2}}{\partial x_{i} \partial x_{i}}$ is the Laplacian operator. Substituting eq.(11) into (15) gives after small algebra 
$\frac{\partial r_{d}}{\partial x_{i}}=\left(\frac{\mu}{3}+\frac{\rho u_{i} h_{m_{i}}}{2}\right)^{-1}\left[\bar{r}_{m_{i}}-\frac{h_{m_{k}}}{2} \frac{\partial r_{m_{i}}}{\partial x_{k}}+\frac{\rho u_{i} h_{m_{i}}}{2} \frac{\partial r_{d}}{\partial x_{i}}-\frac{\delta}{2} \frac{\partial r_{m_{i}}}{\partial t}\right]$ no sum in $i$

where

$$
\bar{r}_{m_{i}}=r_{m_{i}}+\frac{\mu}{3} \frac{\partial r_{d}}{\partial x_{i}}
$$

and $r_{m_{i}}$ is given by eq.(8).

Inserting eq.(16) into eq.(6) gives

$$
r_{d}+c_{i}\left(\bar{r}_{m_{i}}-\frac{h_{m_{k}}}{2} \frac{\partial r_{m_{i}}}{\partial x_{k}}+\frac{\rho u_{i} h_{m_{i}}}{2} \frac{\partial r_{d}}{\partial x_{i}}-\frac{\delta}{2} \frac{\partial r_{m_{i}}}{\partial t}\right)=0 \text { no sum in } i
$$

with

$$
c_{i}=\left(\frac{2 \mu}{3 h_{d_{i}}}+\frac{\rho u_{i} h_{m_{i}}}{h_{d_{i}}}\right)^{-1} \quad \text { no sum in } i
$$

Extracting the pressure terms from the brackets in (18) gives
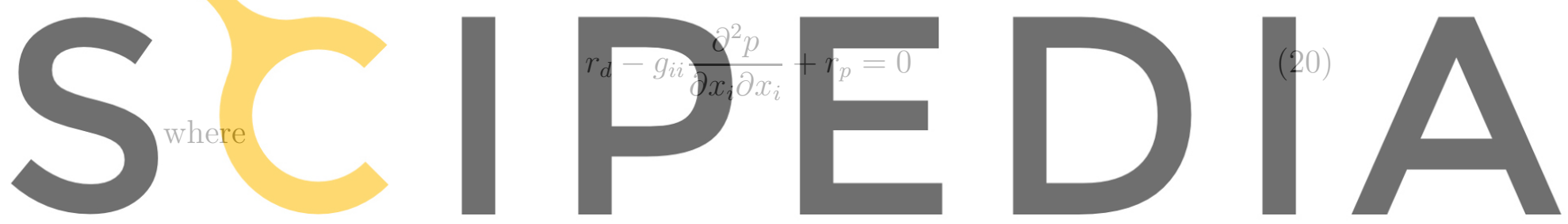

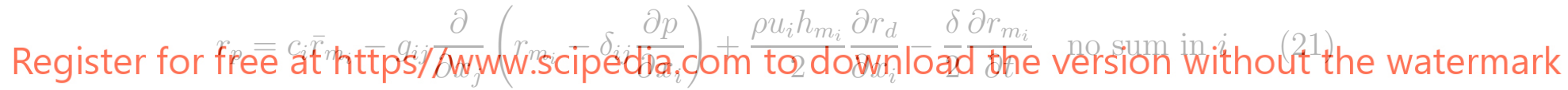

and

$$
g_{i j}=\left(\frac{4 \mu}{3 h_{d_{i}} h_{m_{j}}}+\frac{2 \rho u_{i} h_{m_{i}}}{h_{d_{i}} h_{m_{j}}}\right)^{-1} \text { no sum in } i
$$

Note that for $h_{m_{i}}=h_{m_{j}}=h$ where $h$ is a typical grid dimension (i.e. the average element size), the value of $g_{i i}$ is simply

$$
g_{i i}=\left(\frac{4 \mu}{3 h^{2}}+\frac{2 \rho u_{i}}{h}\right)^{-1}
$$

The stabilization parameter $g_{i i}$ has now the form traditionally used in the GLS formulation for the viscous (Stokes) limit $\left(u_{i}=0\right)$ and the inviscid (Euler) limit $(\mu=0)$ and deduced from ad-hoc extensions of the 1D advective-diffusive problems [20-26]. Note, however, that the general form of the stabilization parameter $g_{i i}$ is extracted here from the general FIC formulation without further extrinsic assumptions.

Indeed, the precise computation of the characteristic length values is crucial for the practical applications of above stabilized expressions. 


\section{Fractional step approach}

The momentum equations (5) are first discretized in time using the following scheme

$$
u_{i}^{n+1}=u_{i}^{n}-\frac{\Delta t}{\rho}\left[\rho \frac{\partial\left(u_{i} u_{j}\right)^{n}}{\partial x_{j}}+\frac{\partial p^{n+1}}{\partial x_{i}}-\frac{\partial \tau_{i j}^{n}}{\partial x_{j}}-b_{i}^{n}-\frac{h_{m_{k}}^{n}}{2} \frac{\partial r_{m_{i}}^{n}}{\partial x_{k}}-\frac{\delta^{n}}{2} \frac{\partial r_{m_{i}}^{n}}{\partial t}\right]
$$

Eq.(23) is now split into the two following equations

$$
\begin{aligned}
u_{i}^{*} & =u_{i}^{n}-\frac{\Delta t}{\rho}\left[\rho \frac{\partial\left(u_{i} u_{j}\right)}{\partial x_{j}}-\frac{\partial \tau_{i j}}{\partial x_{j}}-b_{i}-\frac{h_{m_{k}}}{2} \frac{\partial r_{m_{i}}}{\partial x_{k}}-\frac{\delta}{2} \frac{\partial r_{m_{i}}}{\partial t}\right]^{n} \\
u_{i}^{n+1} & =u_{i}^{*}-\frac{\Delta t}{\rho} \frac{\partial p^{n+1}}{\partial x_{i}}
\end{aligned}
$$

Note that the sum of eqs.(24) and (25) gives the original form of eq.(23).

Substituting eq.(25) into the stabilized mass balance equation (20) gives the standard Laplacian of pressure form
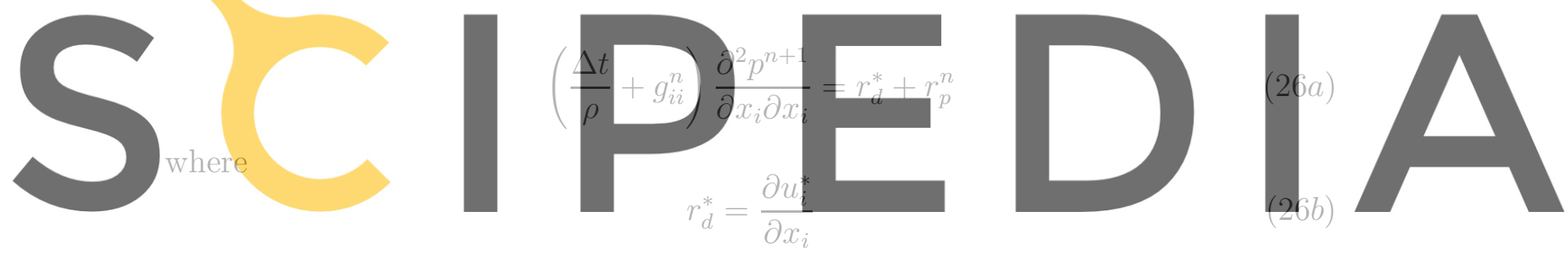

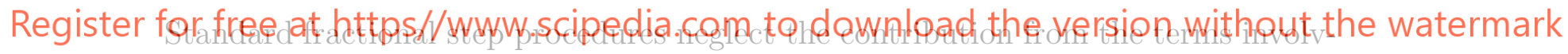

ing $g_{i i}$ in eq. (26). These terms have an additional stabilization effect which improves the numerical solution when the values of $\Delta t$ are small. The influence of the cross derivative terms $\frac{\partial^{2} p}{\partial x_{i} \partial x_{j}}$ kept within $r_{p}$ should be studied in the future.

The stabilized free surface wave equation (7) is discretized in time to give

$$
\beta^{n+1}=\beta^{n}-\Delta t\left[v_{i}^{n+1} \frac{\partial \beta^{n}}{\partial x_{i}}-v_{3}^{n+1}-\frac{h_{\beta_{j}}}{2} \frac{\partial r_{\beta}^{n}}{\partial x_{j}}-\frac{\gamma}{2} \frac{\partial r_{\beta}^{n}}{\partial t}\right] \quad i, j=1,2
$$

A typical solution in time includes the following steps.

Step 1. Solve explicitely for the so called fractional velocities $u_{i}^{*}$ using eq. (24).

Step 2. Solve for the pressure field $p^{n+1}$ solving the laplacian equation (26a). The pressures at the free surface computed from step 6 below in the previous time step are used as boundary conditions for solution of eq.(26b). Alternatively a zero pressure condition at the surface must be imposed if the mesh boundary nodes are updated in a lagrangean manner and placed on the new free surface. 
Step 3. Compute the velocity field $u_{i}^{n+1}$ at the updated configuration for each mesh node using eq. (25)

Step 4. Compute the new position of the free surface elevation $\beta^{n+1}$ in the fluid domain by using eq.(27).

Step 5. Compute the movement of the submerged body by solving the dynamic equations of motion in the body subjected to the pressure field $p^{n+1}$ and the viscous stresses $\tau_{i j}^{n}$.

Step 6. Compute the new position of mesh nodes in the fluid domain at time $n+1$ by using the mesh update algorithm described in next section. The pressure in the free surface is obtained from Benouilli equation as

$$
p^{n+1}=p^{o}+\rho g\left(\beta^{n+1}-\beta^{o}\right)
$$

where $\beta^{\circ}$ and $p^{o}$ are reference values of the free surface elevation and the pressure respectively and $g$ is the gravity constant.

As already mentioned the effect of changes in the free surface elevation can be introduced in the step 2 of the flow solution as a prescribed pressure acting on the Equation (28) does not account for viscosity and rotational effects in the fluid. These
effects are however negligible in the free surface transport process and the pressure
given by eq.(28) is a good approximation. Note that if the mesh is detormed after 4 each time step so that the nodes are placed at the position defined by $\beta^{n+1}$, the use

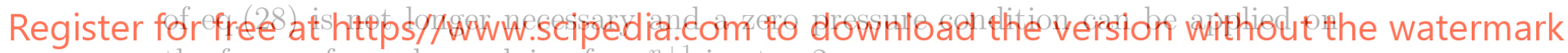
the free surface when solving for $p^{n+1}$ in step 2.

\section{Finite element discretization}

Space discretization is carried out using the finite element method [14]. The stabilized formulation described allows an equal order interpolation of velocities and pressure $[8,14]$. A linear interpolation over four node tetrahedra for both $u_{i}$ and $p$ is chosen in the examples shown in the paper. Similarly linear triangles are chosen to interpolate $\beta$ on the free surface mesh. The velocity and pressure fields are interpolated within each element in the standard finite element manner as

$$
\begin{gathered}
u_{i}=\sum_{j} N_{j}\left(\bar{u}_{i}\right)_{j} \\
p_{i}=\sum_{j} N_{j} \bar{p}_{j}
\end{gathered}
$$

where $N_{j}$ are the linear shape functions interpolating the velocity and pressure fields, respectively and $(\bar{\cdot})$ denote nodal values. 
Similarly the wave height is discretized as

$$
\beta=\sum_{j} N_{\beta_{j}} \bar{\beta}_{j}
$$

where $N_{\beta_{j}}$ are linear shape functions defined over the three node triangles discretizing the free surface.

The discretized integral form in space is obtained by applying the standard Galerkin procedure to eqs.(24),(25),(26a) and (27) and the boundary conditions (13). Solution of the discretized problem follows the pattern given below.

Step 1. Solve for the nodal fractional velocities

$$
\mathbf{u}^{*}=\mathbf{M}^{-1} \mathbf{f}_{1}^{n}
$$

with

$$
\begin{gathered}
M_{i j}=\int_{\Omega} N_{i} N_{j} d \Omega \\
\mathbf{f}^{n}=\left\{\begin{array}{c}
\mathbf{f}_{1} \\
\mathbf{f}_{2} \\
\vdots \\
\mathbf{f}_{n}
\end{array}\right\}^{n}, \quad \mathbf{f}_{k}^{n}=\left\{\begin{array}{c}
f_{k_{1}} \\
f_{k_{2}} \\
f_{k_{1}}
\end{array}\right\}^{n} \\
f_{k_{i}}^{n}=\int_{\Omega} N_{k}\left[u_{i}-\frac{\Delta t}{\rho}\left(\rho \frac{\partial\left(u_{i} u_{j}\right)}{\partial x_{j}}-b_{i}-\frac{h_{m_{k}}}{2} \frac{\partial r_{m_{i}}}{\partial x_{k}}-\frac{\delta}{2} \frac{\partial r_{m_{i}}}{\partial t}\right)\right]^{n} d \Omega+ \\
+\int_{\Omega} \frac{\Delta t}{\rho} \frac{\partial N_{k}}{\partial x_{j}} \tau_{i j}^{n} d \Omega-\int_{\Gamma_{t}} \frac{\Delta t}{\rho} N_{k} t_{i}^{n} d \Gamma \quad, \quad i=1,2,3
\end{gathered}
$$

The solution of eq.(31) can be speeded up by diagonalizing matrix M. Alternatively a simple Jacobi iteration procedure can be used and this has proved to converge in very few iterations.

No boundary condition is applied when solving for the fractional velocities $u_{i}^{*}$ in eq.(31) as these velocities can be interpreted as a predicted value of the actual velocities. The kinematic boundary conditions (14) are applied in step 3 as shown below.

Step 2. Solve for the nodal pressures at time $n+1$

$$
\begin{gathered}
\mathbf{H p}^{n+1}=\mathbf{q}^{n} \\
H_{k l}=\int_{\Omega} \frac{\partial N_{k}}{\partial x_{i}}\left(\frac{\Delta t}{\rho}+g_{i i}^{n}\right) \frac{\partial N_{l}}{\partial x_{i}} d \Omega
\end{gathered}
$$




$$
q_{k}^{n}=\int_{\Omega} \frac{\partial N_{k}}{\partial x_{i}} u_{i}^{*} d \Omega-\int_{\Omega} N_{k} r_{d}^{n} d \Omega+\int_{\Gamma} N_{k} \frac{\rho}{\Delta t}\left(\frac{\Delta t}{\rho}+g_{i i}^{n}\right) u_{i}^{n} n_{i} d \Gamma
$$

Note that for simplicity the velocities $u_{i}^{n+1}$ in the boundary integral of eq.(40) have been substituted by $u_{i}^{n}$. This integral can be neglected in solid walls and stationary free surfaces where the normal velocity is zero.

Recall that the pressures computed from step 6 are used as boundary condition for solution of eq.(38). If the mesh boundary nodes are updated then a zero pressure condition must be applied at the free surface nodes.

Step 3. Solve for the nodal velocities at time $n+1$

$$
\mathbf{u}^{n+1}=\mathbf{M}^{-1} \overline{\mathbf{f}}^{n+1}
$$

where $\mathbf{M}$ is given by (35) and

$$
\bar{f}_{k_{i}}^{n+1}=\int_{\Omega} N_{k}\left[u_{i}^{*}-\frac{\Delta t}{\rho} \frac{\partial p^{n+1}}{\partial x_{i}}\right] d \Omega
$$

The kinematic boundary conditions on the nodal velocities (eq.(14)) are imposed when solving eq.(41).

\section{Step 4. Solve for the new free surface heights at the time $n+1$}

The new free surface elevation $\beta^{n+1}$ in the fluid domain is computed as

$$
\boldsymbol{\beta}^{n+1}=\mathbf{M}_{\beta}^{-1} \mathbf{s}^{n+1}
$$

with

$$
\begin{gathered}
\mathbf{M}_{\beta}=\int_{\Gamma_{\beta}} \mathbf{N}_{\beta}^{T} \mathbf{N}_{\beta} d \Gamma \\
s_{i}^{n+1}=\int_{\Gamma} N_{\beta_{i}}\left[\beta^{n}-\Delta t\left(v_{k}^{n+1} \frac{\partial \beta^{n}}{\partial x_{k}}-v_{3}^{n+1}-\frac{\gamma}{2} \frac{\partial r_{\beta}^{n}}{\partial t}\right)\right] d \Gamma+\int_{\Gamma} \frac{h_{\beta_{j}}}{2} \frac{\partial N_{\beta_{i}}}{\partial x_{j}} r_{\beta} d \Gamma
\end{gathered}
$$

In the derivation of eq.(42) the assumption that $r_{\beta}=0$ at the contours of the free surface domain has been made.

Steps 5 and 6 follow the process described in previous section. 


\section{Computation of the stabilization parameters}

Accurate evaluation of the stabilization parameters is one of the crucial issues in stabilized methods. Most of existing methods use expressions which are direct extensions of the values obtained for the simplest 1D case. It is also usual to accept the so called SUPG assumption, i.e. to admit that vector $\mathbf{h}_{m}$ has the direction of the velocity field. This unnecessary restriction leads to instabilities when sharp layers transversal to the velocity direction are present. This additional defficiency is then corrected by adding a shock capturing or crosswind stabilization term [10].

Let us first assume for simplicity that the stabilization parameters for the mass balance equations are the same than those for the momentum equations. This implies

$$
\mathbf{h}_{m}=\mathbf{h}_{d}
$$

The problem remains now finding the value of the characteristic length vectors $\mathbf{h}_{m}$. Indeed, the components of $\mathbf{h}_{m}$ can introduce the necessary stabilization along the streamline and transversal directions to the flow.

Excellent results have been obtained in all examples using linear tetrahedra and a different value of the characteristic length vector for each momentum equation defined by

$$
\mathbf{h}_{m_{i}}=h_{s} \frac{\mathbf{u}}{|\mathbf{u}|}+h_{c} \frac{\boldsymbol{\nabla} u}{|\boldsymbol{\nabla} u|} \quad i=1,2,3
$$

where $u=|\mathbf{u}|$ and $h_{s}$ and $h_{c}$ are the "streamline" and "cross wind" contributions given by

$$
\begin{aligned}
h_{s} & =\max \left(\mathbf{l}_{j}^{T} \mathbf{u}\right) /|\mathbf{u}| \\
h_{c} & =\max \left(\mathbf{l}_{j}^{T} \boldsymbol{\nabla} u\right) /|\boldsymbol{\nabla} u| \quad, \quad j=1, n_{s}
\end{aligned}
$$

where $\mathbf{l}_{j}$ are the vectors defining the element sides $\left(n_{s}=6\right.$ for tetrahedra).

An alternative method for computing vector $\mathbf{h}_{m}$ in a more consistent manner is explained in next section.

As for the free surface equation the following value of the characteristic length vector $\mathbf{h}_{\beta}$ has been taken

$$
\mathbf{h}_{\beta}=\bar{h}_{s} \frac{\mathbf{u}}{|\mathbf{u}|}+\bar{h}_{c} \frac{\boldsymbol{\nabla} \beta}{|\boldsymbol{\nabla} \beta|}
$$

The streamline parameter has been obtained by eq.(45) using the value of the velocity vector $\mathbf{u}$ over the 3 node triangles discretizing the free surface and $n_{s}=3$.

The cross wind parameter has been computed by

$$
\bar{h}_{c}=\max \left[\mathbf{l}_{j}^{T} \boldsymbol{\nabla} \beta\right] \frac{1}{|\boldsymbol{\nabla} \beta|} \quad, \quad j=1,2,3
$$


Note that the cross-wind terms in eqs.(44) and (47) account for the effect of the gradient of the solution in the stabiliztion parameters. This is an standard assumption in most "shock-capturing" stabilization procedures [34-37].

Regarding the time stabilization parameters $\delta$ and $\gamma$ in eqs.(5) and (7) the value $\delta=\gamma=\Delta t$ has been taken for solution of the examples presented in the paper. A more consistent evaluation following the dimishing residual technique described next is described in [7] for transient advective-diffusive problems.

\subsection{Computation of the characteristic length parameters via a diminishing residual procedure}

The idea of this technique first presented in [4] and tested in [5-7] for advectivediffusive problems is the following. Let us assume that a finite element solution for the velocity and pressure fields has been found for a given mesh. The point wise residual of the momentum equation corresponding to this particular solution is (assuming $\delta=0$ in eq.(5))

$$
{ }^{1} r_{m_{i}}=r_{m_{i}}-\frac{1}{2} h_{m_{j}} \frac{\partial r_{m_{i}}}{\partial x_{j}}
$$

The average residual over an element can be defined as

$$
{ }^{1} r_{m_{i}}^{(e)}=\frac{1}{\Omega^{(e)}} \int_{\Omega^{(e)}}{ }^{1} r_{m_{i}} d \Omega
$$

Let us assume now that an enhanced numerical solution has been found for the same mesh and the same approximation (i.e. neither the number of elements nor the element type have been changed). This enhanced solution could be based, for instance, in a superconvergent recovery of derivatives [14,38,39]. The element residual for the enhanced solution is denoted ${ }^{2} r_{m_{i}}^{(e)}$. As the element residuals must tend to zero, the following condition must be satisfied

$$
{ }^{1} r_{m_{i}}^{(e)}-{ }^{2} r_{m_{i}}^{(e)} \geq 0
$$

Above equation applies for ${ }^{1} r_{m_{i}}^{(e)}>0$. Clearly for ${ }^{1} r_{m_{i}}^{(e)}<0$ the inequality in eq. $(51)$ should be changed to $\leq 0$.

Eq. (54) provides a system of equations which unkowns are the characteristic length parameters. Substituting eq.(49) into (51) and appling the identity condition in eq.(51) gives

$$
\mathbf{h}_{m}^{(e)}=\mathbf{A}^{-1} \mathbf{f}
$$


with

$$
\begin{aligned}
A_{i j} & =2\left[\frac{{ }^{2} \partial r_{m_{i}}^{(e)}}{\partial x_{j}}-\frac{{ }^{1} \partial r_{m_{i}}^{(e)}}{\partial x_{j}}\right] \\
f_{i} & ={ }^{2} r_{m_{i}}^{(e)}-{ }^{1} r_{m_{i}}^{(e)}
\end{aligned}
$$

The following "adaptive" algorithm can be proposed for obtaining a stabilized solution:

1. Solve for numerical values of velocities and pressure for an initial value $\mathbf{h}_{m}^{(e)}=$ $\mathbf{h}_{o}^{(e)}$. Compute ${ }^{1} r_{m_{i}}^{(e)}$.

2. Evaluate the enhanced velocity and pressure fields. Compute ${ }^{2} r_{m_{i}}^{(e)}$.

3. Compute the updated value of $\mathbf{h}_{m}^{(e)}$ using eq.(52).

4. Repeat (1)-(3) until a stable solution is found.

Above strategy can be naturally incorporated into a transient solution scheme where the value of $\mathbf{h}_{m}^{(e)}$ is updated after the solution for each time step has been found.

The assumption $\mathbf{h}_{d}=\mathbf{h}_{m}$ can be relaxed and an independent value of the characteristic length vector $\mathbf{h}_{d}$ for the mass balance equation can be found following a similar approach as described for computing $\mathbf{h}_{m}$. Further details can be found in [2-6] where this technique has been successfully tested for steady state and transient advective-diffusive problems.

\section{A simple algorithm for updating the mesh nodes}

Different techniques have been proposed for dealing with mesh updating in fluidstructure interaction problems. The general aim of all methods is to prevent element distorsion during mesh deformation [44,48].

Chiandussi, Bugeda and Oñate [13] have recently proposed a simple method for movement of mesh nodes ensuring minimum element distorsion. The method is based on the iterative solution of a fictitious linear elastic problem on the mesh domain. In order to minimize mesh deformation the "elastic" properties of each mesh element are appropiately selected so that elements suffering greater movements are stiffer. The basis of the method is given below.

Let us consider an elastic domain with homogeneous isotropic elastic properties characterized by the Young modulus $\bar{E}$ and the Poisson coefficient $\nu$. Once a discretized finite element problem has been solved using, for instance, standard $C^{o}$ linear triangles (in 2D) or linear tetraedra (in 3D), the principal stresses ${ }^{1} \sigma_{i}$ at the center of each element are obtained as

$$
{ }^{1} \sigma_{i}=\bar{E}\left[\varepsilon_{i}-\nu\left(\varepsilon_{j}+\varepsilon_{k}\right)\right] \quad i, j=1,2,3 \text { for } 3 \mathrm{D}
$$


where $\varepsilon_{i}$ are the principal strains.

Let us assume now that a uniform strain field $\varepsilon_{i}=\bar{\varepsilon}$ throughout the mesh is sougth. The principal stresses are then given by

$$
{ }^{2} \sigma_{i}=E \bar{\varepsilon}(1-2 \nu) \quad i=1,2,3 \text { for } 3 \mathrm{D}
$$

where $E$ is the unknown Young modulus for the element.

A number of criteria can be now used to find the value of $E$. The most effective approach found in [13] is to equal the element strain energies in both analysis. Thus

$$
\begin{aligned}
U_{1} & ={ }^{1} \sigma_{i} \varepsilon_{i}=\bar{E}\left[\left(\varepsilon_{1}^{2}+\varepsilon_{2}^{2}+\varepsilon_{3}^{2}\right)-2 \nu\left(\varepsilon_{1} \varepsilon_{2}+\varepsilon_{2} \varepsilon_{3}+\varepsilon_{1} \varepsilon_{3}\right)\right] \\
U_{2} & ={ }^{2} \sigma_{i} \varepsilon_{i}=3 E \bar{\varepsilon}^{2}(1-2 \nu)
\end{aligned}
$$

Equaling eqs.(57) and (58) gives the sought Young modulus E as

$$
E=\frac{\bar{E}}{3 \bar{\varepsilon}^{2}(1-2 \nu)}\left[\left(\varepsilon_{1}^{2}+\varepsilon_{2}^{2}+\varepsilon_{3}^{2}\right)-2 \nu\left(\varepsilon_{1} \varepsilon_{2}+\varepsilon_{2} \varepsilon_{3}+\varepsilon_{1} \varepsilon_{3}\right)\right]
$$

Note that the element Young modulus is proportional to the element deformation as desired. Also recall that both $\bar{E}$ and $\bar{\varepsilon}$ are constant for all elements in the mesh. The solution process includes the following two steps.

Step 1. Consider the finite element mesh as a linear elastic solid with homogeneous material properties characterized by $\bar{E}$ and $\nu$. Solve the corresponding elastic problem with imposed displacements at the mesh boundary. These displacements can be due to a prescribed motion of a body within a fluid, to changes in the shape of the domain in an optimum design problem, etc.

Step 2. Compute the principal strains and the values of the new Young modulus in each element using eq.(59) for a given value of $\bar{\varepsilon}$. Repeat the finite element solution of the linear elastic problem with prescribed boundary displacements using the new values of $E$ for each element.

The movement of the mesh nodes obtained in the second step ensures a quasi uniform mesh distorsion. Further details on this method including other alternatives for evaluating the Young modulus $E$ can be found in [8].

The previous algorithm for movement of mesh nodes is able to treat the movement of the mesh due to changes in position of fully submerged and semi-submerged bodies. Note however that if the floating body intersects the free surface, the changes in the analysis domain geometry can be very important. From one time step to other emersion or inmersion of significant parts of the body can occur.

A posible solution to this problem is to remesh the analysis domain. However for most problems, a mapping of the moving surfaces linked to mesh updating algorithm described above can avoid remeshing (Figure 2). 
The surface mapping technique used in this work is based on transforming 3D curved surfaces into reference planes. This allows to compute within each plane the local (in-plane) coordinates of the nodes for the final surface mesh accordingly to the changes in the floating line. The final step is to transform back the local coordinates of the surface mesh in the reference plane to the final curved configuration which incorporates the new floating line [3].

\section{$7 \quad$ Examples}

\subsection{Example 1. Square cavity problem}

The purpose of this example is to test the stabilized formulation presented in the solution of a standard benchmark problem solved by a number of authors $[22,23,40,41]$. Figure 3 shows the definition of the problem solved with an unstructured mesh of 3000 linear tetrahedra for a Reynolds number value of 1 .

The steady-state solution was sought using the fractional step algorithm previously described. Results in Figure 3 are tabulated for the horizontal velocity along the vertical centerline of the mid-section and for vertical velocity and pressure along the horizontal centerline is of the same section. Numerical results are fully stable and agree well with similar solutions reported in the mentioned reference.

\subsection{Example 2. Submerged NACA 0012 profile}

A 2D submerged NACA0012 profile at $\alpha=5^{\circ}$ angle of attack is studied. This configuration was tested experimentally by Duncan [42] for high Reynolds numbers $(\mathrm{Re}=400000)$ and modelled numerically using the Euler equations by several authors [43-45]. The submerged depth of the airfoil is equal to the chord and this was used as the length $(\mathrm{L})$ for normalizing the problem. The Froude number for all the cases tested was set to $F r=\frac{u}{\sqrt{g L}}=0.5672$ where $u$ is the incoming flow velocity at infinity.

The stationary free surface and the pressure distribution in the domain are shown in Figure 4. The non-dimensional wave heights compare well with the experimental results of $[42]$.

\subsection{Example 3. Sphere falling in a tube filled with liquid}

The movement of a sphere falling by gravity in a cylindrical tube filled with liquid is studied. The relationship between the diameters of the sphere and the tube is 1:4. The Reynolds number for the stationary speed is 100 . The mesh has 85765 element with 13946 nodes (Figure 5).

Figures 6 and 7 show the mesh deformation and contours of the mesh deformation and of the velocity in the domain for different times, respectively. The evolution of 
the falling speed is shown in Figure 8. Note the good agreement with the so called Stokes velocity computed by equaling the weight of the sphere with the resistance to the movement of the sphere expressed in terms of the velocity. Obviously, this value is slightly greater than the actual one as frictional effects are neglected.

A similar problem for a much greater number of spheres has been solved by Johnson and Tezduyar [47].

\subsection{Example 4. Movement of a submerged sphere in an open channel}

Figure 9 shows the geometry of the channel and the position of the sphere of $2 \mathrm{~m}$ diameter with a weight of $1000 \mathrm{~N}$ and a rotational inertia of $1000 \mathrm{kgm}^{2}$. A mesh of 19870 linear tetrahedra with 4973 nodes has been used for the analysis.

The problem has been analyzed for values of Reynolds number $=200$ and Froude number $=0.71$ corresponding to a velocity of $1 \mathrm{~m} / \mathrm{s}$ at the inlet.

It is assumed that the sphere can only move vertically and rotate around the global $y$ axes due to the forces induced by the fluid. The vertical displacement is constrained by a spring linking the sphere to the ground. An initial vertical velocity of $1 \mathrm{~m} / \mathrm{s}$ for the sphere has been taken.

Figure 10 shows a plot of the time evolution of the vertical displacement of the sphere. The contours of the velocity module in the fluid on two perpendicular planes at different times is shown in Figure 11. the deformation of the free surface at $t=0.47 \mathrm{~s}$. and $3.16 \mathrm{~s}$. is shown in Figure 12.

\subsection{Example 5. Interactions of a rigid vertical cylinder with a moving stream}

The definition of the problem is clearly seen in Figure 13. The cylinder diameter is $2 \mathrm{~m}$ and the stream speed is $1 \mathrm{~m} / \mathrm{s}$. The Froude and Reynolds numbers are 1.0 and 200, respectively. The walls of the cylinder are assumed to be rigid in this case. A mesh of 35567 tetrahedra and 4670 nodes is used for the analysis.

Figure 14 shows the contours of the velocity module and the vertical displacement in the mesh for a time $t=4.57 \mathrm{~s}$. Note the important deformation of the free surface in this problem.

\subsection{Example 6. Wigley hull}

The last case considered here is the well known Wigley Hull, given by the analytical formula $y=0.5 B\left(1-4 x^{2}\right)\left(1-z^{2} / D^{2}\right)$ where $B$ and $D$ are the beam and the draft of the ship hull at still water. 


\begin{tabular}{|c|c|c|}
\hline & Experimental & Numerical \\
\hline Test 1 & $5.210^{-3}$ & $4.910^{-3}$ \\
\hline Test 2 & $5.210^{-3}$ & $5.310^{-3}$ \\
\hline Test 3 & $4.910^{-3}$ & $5.110^{-3}$ \\
\hline
\end{tabular}

Table 1. Wigley Hull. Total resistance coefficient

The same configuration was tested experimentally in [46] and modelled numerically by several authors [43-45]. We use here a non structured 3D finite element mesh of 65434 linear tetrahedra, with a reference surface of 7800 triangles, partially represented in Figure 15.

Figure 15 also shows the viscous analysis of the Wigley model in three different cases $\left(L_{p p}=6 m, F_{n}=0.316, \mu=10^{-3} \mathrm{Kg} / \mathrm{m} . \mathrm{s}\right)$. In the first case the volume mesh was considered fixed, not allowing free surface nor ship movements. Secondly the volume mesh was updated due to free surface movement, considering the model fixed. The third case corresponds to the analysis of a real free model including the mesh updating due to free surface evaluation and ship movement (sinkage and trim). A Smagorinsky turbulence model was used in all the cases.

Table 1 shows the obtained total resistance coefficient in the three cases studied compared with the experimental data.

In the study of the free model the numerical values of sinkage and trim were $-0.1 \%$ and 0.035 , respectively, while experiment gave $-0.15 \%$ and 0.04 .

Figure 15 whows the pressure distribution obtained near the Wigley hull in the case of the free model. The free surface in transparent allowing seeing some streamlines. The obtained mesh deformation in this case is also presented.

Comparisons of the obtained body wave profile with the experimental data for the free and fixed models are shownin Figure 15. Significant differences are found close to stern in the case of the fixed model.

\section{Conclusions}

The finite increment calculus method allows to derive stabilized forms of the governing differential equations for a viscous fluid with a free surface. Solution of the new stabilized equations written in ALE form with a semi-implicit fractional step finite element method provides a straight-forward algorithm for fluid-structure interaction analysis.

The mesh-moving scheme presented ensures minimum mesh distorsion for large mesh displacements. The formulation presented is adequate for solving large scale fluidstructure interaction problems in naval architecture and offshore engineering. 


\section{Acknowledgements}

Support for this work was provided by the European Community through projects Brite-Euram BR 967-4342 SHEAKS and Esprit 24903 FLASH.

This support of Empresa Nacional BAZAN de Construcciones Navales y Militares, S.A. is also gratefully acknowledged.

Thanks are also given to Mr. J. Royo for his help in computing some of the examples presented. 


\section{References}

[1] E. Oñate and J. García, A stabilized finite element method for analysis of fluidstructure interaction problems involving the surface waves, in Computational Methods for Fluid-Structure Interaction, T. Kvamsdal et al., (Eds.), TAPIR Publishers, Norway, 1999.

[2] E. Oñate and J. García, "A methodology for analysis of fluid-structure interaction accounting for free surfacw waves", European Conference on Computational Mechanics (ECCM99), August 31-September 3, Munich, Germany, 1999.

[3] J. García, "A finite element method for analysis of naval structures" (in Spanish), Ph.d. Thesis, Univ. Politeècnica de Catalunya, December 1999.

[4] E. Oñate, "Derivation of stabilized equations for advective-diffusive transport and fluid flow problems", Comput. Meth. Appl. Mech. Engng., Vol. 151, 1-2, pp. 233-267, 1998.

[5] E. Oñate, J. García and S. Idelsohn Computation of the stabilization parameter for the finite element solution of advective-diffusive problems Int. J. Num. Meth. Fluids, Vol. 25, pp. 1385-1407, 1997.

[6] E. Oñate, J. García and S. Idelsohn, An alpha-adaptive approach for stabilized finite element solution of advective-diffusive problems with sharp gradients, New Adv. in Adaptive Comp. Met. in Mech., P. Ladeveze and J.T. Oden (Eds.), Elsevier, 1998.

[7] E. Oñate and M. Manzan, A general procedure for deriving stabilized space-time finite element methods for advective-diffusive problems, Publication PI-133, CIMNE, Barcelona, July 1998.

[8] E. Oñate, A finite element method for incompressible viscous flows using a finite increment calculus formulation, Research Report N. 150, CIMNE, Barcelona, January 1999.

[9] E. Oñate and S. Idelsohn, A mesh free finite point method for advective-diffusive transport and fluid flow problems,, Computational Mechanics, 21, 283-292, 1988.

[10] E. Oñate, C. Sacco and S. Idelsohn, "A finite point method for incompressible flow problems", Publication CIMNE PI-167, July 1999. To be published in ????

[11] J. García, E. Oñate, H. Sierra, C. Sacco and S. Idelsohn, "A stabilized numerical method for analysis of ship hydrodynamics", ECCOMAS98, K. Papaliou et al. (Eds.), J. Wiley, 1998.

[12] E. Oñate, S. Idelsohn, C. Sacco and J. García, "Stabilization of the numerical solution for the free surface wave equation in fluid dynamics", ECCOMAS98, K. Papalion et al. (Eds.), J. Wiley, 1998.

[13] G. Chiandusi, G. Bugeda and E. Oñate, A simple method for update of finite element meshes, Research Report 147, CIMNE, Barcelona, January 1999. 
[14] O.C. Zienkiewicz and R.C. Taylor, The finite element method, 4th Edition, Vol. 1, McGraw Hill, 1989, Vol. 2, McGraw Hill, 1991.

[15] R. Codina, A finite element model for incompressible flow problems, Ph.D. Thesis, Univ. Politécnica de Catalunya, Barcelona, June 1992.

[16] A. Brooks and T.J.R. Hughes, "Streamline upwind/Petrov-Galerkin formulation for convection dominated flows with particular emphasis on the incompressible NavierStokes equations", Comput. Meth. Appl. Mech. Engng, 32, 199-259, 1982.

[17] T.J.R. Hughes and M. Mallet, "A new finite element formulations for computational fluid dynamics: III. The generalized streamline operator for multidimensional advective-diffusive systems", Comp. Meth. Appl. Mech. Engng., 58, pp. 305-328, 1986.

[18] P. Hansbo and a. Szepessy, "A velocity-pressure streamline diffusion finite element method for the incompressible Navier-Stokes equations", Comp. Meth. Appl. Mech. Engng., 84, 175-192, 1990.

[19] T.J.R. Hughes, L.P. Franca and M. Balestra, "A new finite element formulation for computational fluid dynamics. V Circumventing the Babuska-Brezzi condition: A stable Petrov-Galerkin formulation of the Stokes problem accomodating equal order interpolations", Comp. Meth. Appl. Mech. Engng., 59, 85-89, 1986.

[20] L.P. Franca and S.L. Frey, "Stabilized finite element methods: II. The incompressible Navier-Stokes equations", Comput. Meth. Appl. Mech. Engn, Vol. 99, pp. 209-233, 1992.

[21] T.J.R. Hughes, G. Hauke and K. Jansen, "Stabilized finite element methods in fluids: Inspirations, origins, status and recent developments", in: Recent Developments in Finite Element Analysis. A Book Dedicated to Robert L. Taylor, T.J.R. Hughes, E. Oñate and O.C. Zienkiewicz (Eds.), (International Center for Numerical Methods in Engineering, Barcelona, Spain, pp. 272-292, 1994.

[22] M.A. Cruchaga and E. Oñate, "A finite element formulation for incompressible flow problems using a generalized streamline operator", Computer Methods in Applied Mechanics and Engineering, 143, 49-67, 1997.

[23] M.A. Cruchaga and E. Oñate, "A generalized streamline finite element approach for the analysis of incompressible flow problems including moving surfaces", Computer Methods in Applied Mechanics and Engineering, 173, 241-255, 1999.

[24] T.J.R. Hughes, L.P. Franca and G.M. Hulbert, "A new finite element formulation for computational fluid dynamics: VIII. The Galerkin/least-squares method for advectivediffusive equations", Comput. Meth. Appl. Mech. Engng., 73, pp. 173-189, 1989.

[25] T.E. Tezduyar, S. Mittal, S.E. Ray and R. Shih, "Incompressible flow computations with stabilized bilinear and linear equal order interpolation velocity-pressure elements", Comp. Meth. Appl. Mech. Engng., 95, 221-242, 1992.

[26] O.C. Zienkiewicz and R. Codina, "A general algorithm for compressible and incompressible flow. Part I: The split characteristic based scheme", Int. J. Num. Meth. in Fluids, 20, 869-85, (1995). 
[27] O.C. Zienkiewicz, K. Morgan, B.V.K. Satya Sai, R. Codina and M. Vázquez, "A general algorithm for compressible and incompressible flow. Part II: Tests on the explicit form", Int. J. Num. Meth. in Fluids, 20, No. 8-9, 886-913, 1995.

[28] T.J.R. Hughes, "Multiscale phenomena: Greens functions, subgrid scale models, bubbles and the origins of stabilized methods", Comput. Meth. Appl. Mech. Engng, Vol. 127, pp. 387-401, 1995.

[29] R. Codina, "A stabilized finite element method for generalized stationary incompressible flows", Publication PI-148, CIMNE, Barcelona, February 1999.

[30] R. Codina and J. Blasco, "Stabilized finite element method for the transient NavierStokes equations based on a pressure gradient operator". To appear in Computer Methods in Appl. Mech. Engng.

[31] F. Brezzi, M.O. Bristeau, L.P. Franca, M. Mallet and G. Rogé, "A relationship between stabilized finite element methods and the Galerkin method with bubble functions", Comput. Meth. Appl. Mech. Engn., Vol. 96, pp. 117-129, 1992.

[32] F. Brezzi, D. Marini and A. Russo, "Pseudo residual-free bubbles and stabilized methods", Computational Methods in Applied Sciences '96, J. Periaux et. al. (Eds.), J. Wiley, 1996.

[33] F. Brezzi, L.P. Franca, T.J.R. Hughes and A. Russo, " $b=\int g$ ", Comput. Meth. Appl. Mech. Engn., 145, 329-339, 1997.

[34] S. Mittal and T.E. Tezduyar, "Massive parallel finite element computation of incompressible flows involving fluid-body interaction", Comp. Meth. Appl. Mech. Engng., 112, 253-282, 1994.

[35] T.J.R. Hughes and M. Mallet, "A new finite element formulations for computational fluid dynamics: IV. A discontinuity capturing operator for multidimensional advectivediffusive system, Comput. Meth. Appl. Mech. Engng., 58, 329-336, 1986.

[36] A.C. Galea a and E.G. Dutra do Carmo, "A consistent approximate upwind PetrovGalerkin method for convection dominated problems", Comput. Meth. Appl. Mech. Engng., 68, 83-95, 1988.

[37] R. Codina, "A discontinuity-capturing crosswind dissipation for the finite element solution of the convection-diffusion equation", Comput. Meth. Appl. Mech. Engng., 110, 325-342, 1993.

[38] R. Codina, "Comparison of some finite element methods for solving the diffusionconvection-reaction equation". To appear in Comp. Meth. Appl. Mech. Engng.

[39] R. Codina, "On stabilized finite element methods for linear systems of convectiondiffusion-reaction equation", Publication CIMNE PI-162, December 1997. To appear in Computer Meth. Appl. Mech. Engng.

[40] M. Fortin and F. Thomasset, "Mixed finite element methods for incompressible flow problems", J. Comput. Phys., 31, 113-145, 1979. 
[41] M. Bercovier and M. Engelman, "A finite element for the numerical solution of viscous incompressible flows", J. Comput. Phys., 30, 181-201, 1979.

[42] J.H. Duncan, "The breaking and non-breaking wave resistance of a two-dimensional hydrofoil", J. Fluid Mech., Vol. 126, 1983.

[43] S. Idelsohn, E. Oñate and J. Garcia, "Finite element solution of free surface ship-wave problem", Int. J. Num. Meth. Engng., 45, 503-508, 1999.

[44] R. Löhner, C. Yang, E. Oñate and S. Idelsohn, "An unstructured grid-based parallel free surface solver", Appl. Num. Math., 31, 271-293, 1999.

[45] E. Oñate, "On the stabilization of numerical solution for advective-diffusive transport and fluid flow problems", Research Report No. 81, CIMNE, Barcelona, 1996. Comp. Meth. Appl. Mech. Engng., 151, 1-2, 233-67, 1998.

[46] Procedings 2nd DTNSRDC Workshop on Ship Wave Resistance Computations. David Taylor Naval Ship Research and Development Center. Noblese, F. and McCarthy J.H. (Eds.), Maryland, USA, 1983.

[47] A.A. Johnson and T.E. Tezduyar, "Simulation of multiple spheres in a liquid-filled tube", Univ. of Minnesota, Supercomputer Institute Report. UMSI 95/212, October 1995. Also in Computer Meth. Appl. Mech. Engng.,1996 
Figure 1. Equilibrium of fluxes in a finite balance domain 

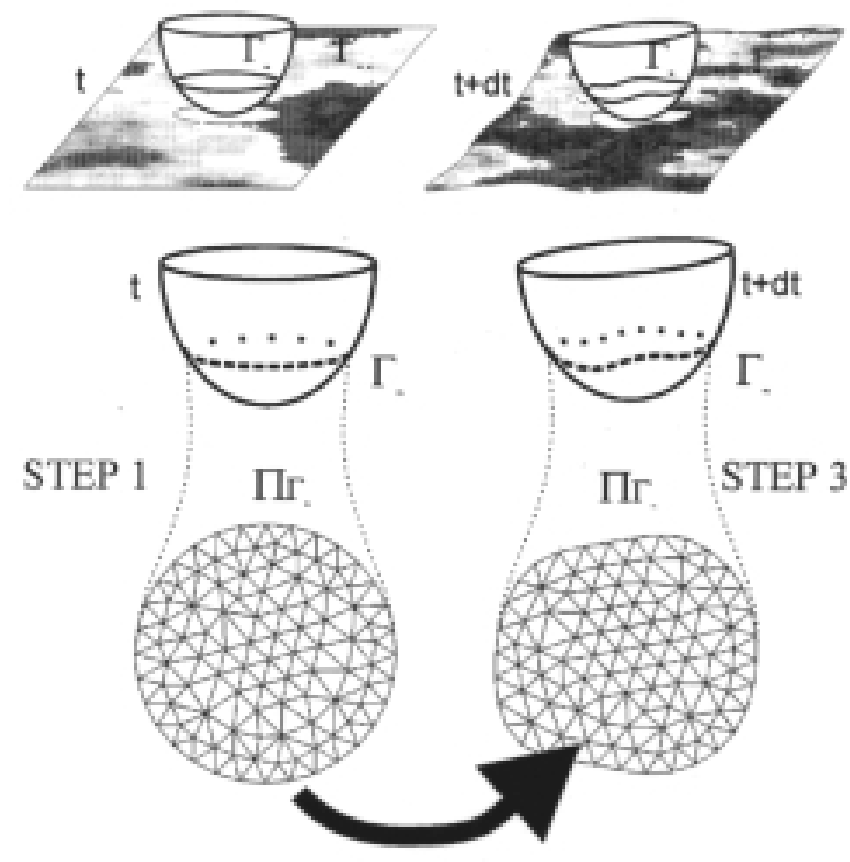

STEP 2

Figure 2. Changes in the fluid interface in a floating body 
Figure 3. Square cavity problem. Problem definition and mesh of 3000 linear tetrahedra. Velocity field for $R e=1$. Distribution of pressure and vertical velocity along horizontal centerline of mid-section and horizontal velocity along vertical centerline 


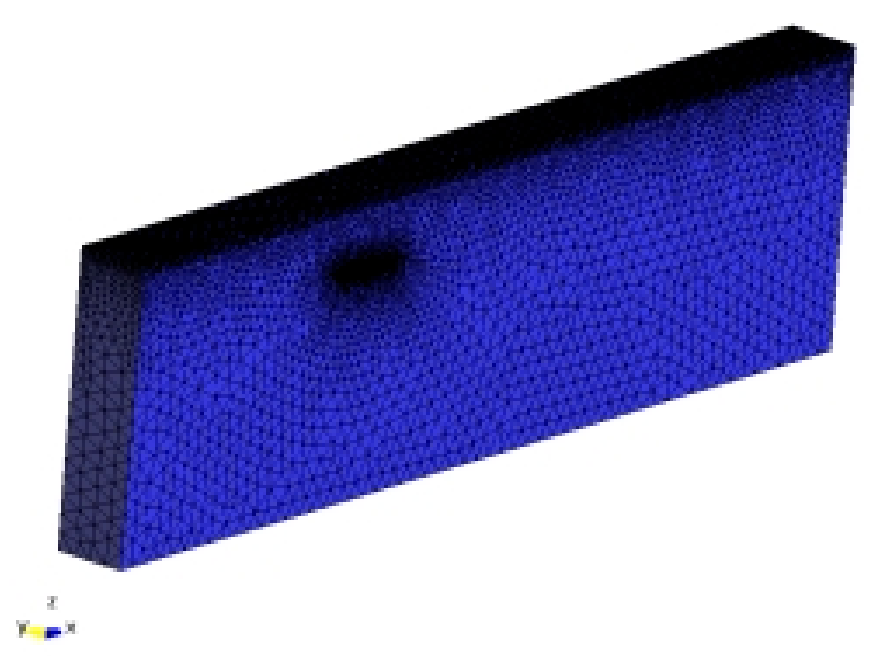

(a)

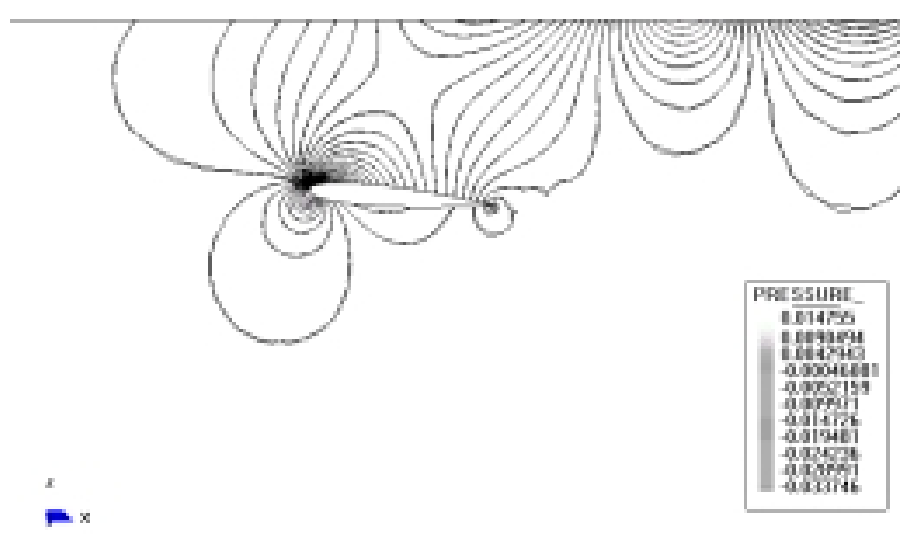

(b)

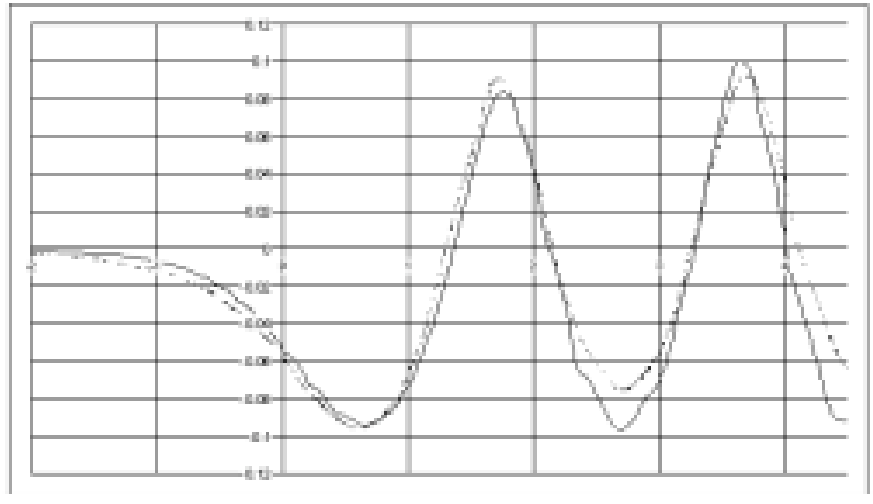

Figure 4. Submerged NACA0012. Mesh of 70000 linear tetrahedra chosen. (a) Pressure contours, (b) Stationary. — present work. - - - experimental values [42] 
Figure 5. Sphere falling in a tube filled with liquid. Detail of the mesh of 85765 linear tetrahedra chosen 
Figure 6. Sphere falling in tube. Sphere position and mesh deformation at different times during the falling process 
(a)

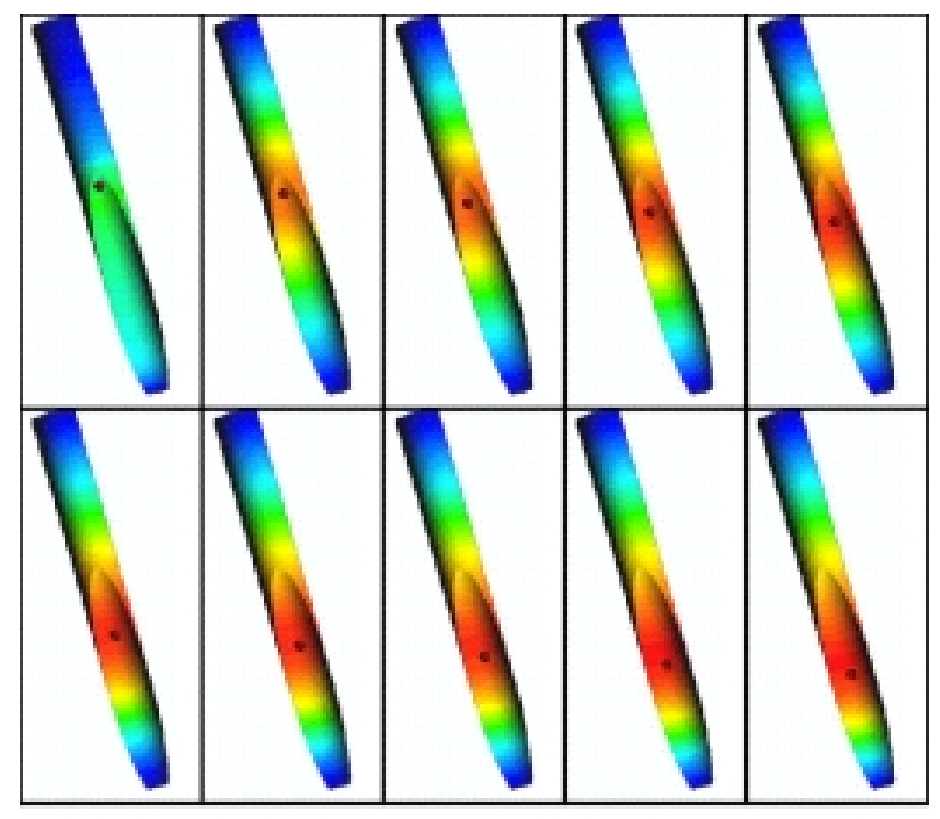

(b)

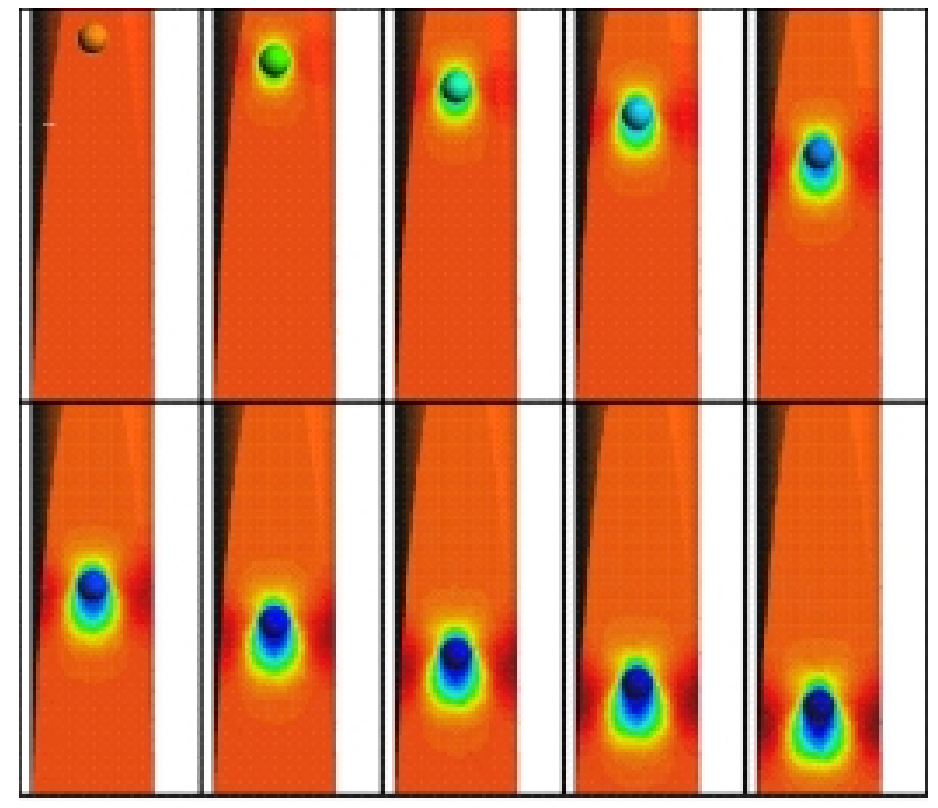

Figure 7. Falling sphere. Evolution of contours of the mesh deformation (a) and the velocity module (b) 


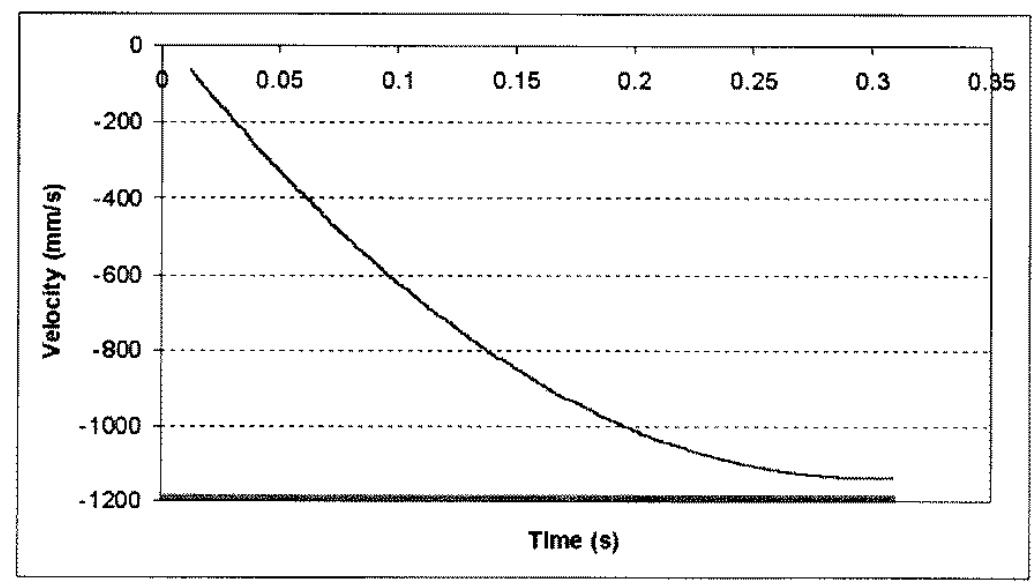

Figure 8. Falling sphere. Evolution of the falling speed. Straight line indicates the Stokes speed $(1,195 \mathrm{~m} / \mathrm{s})$

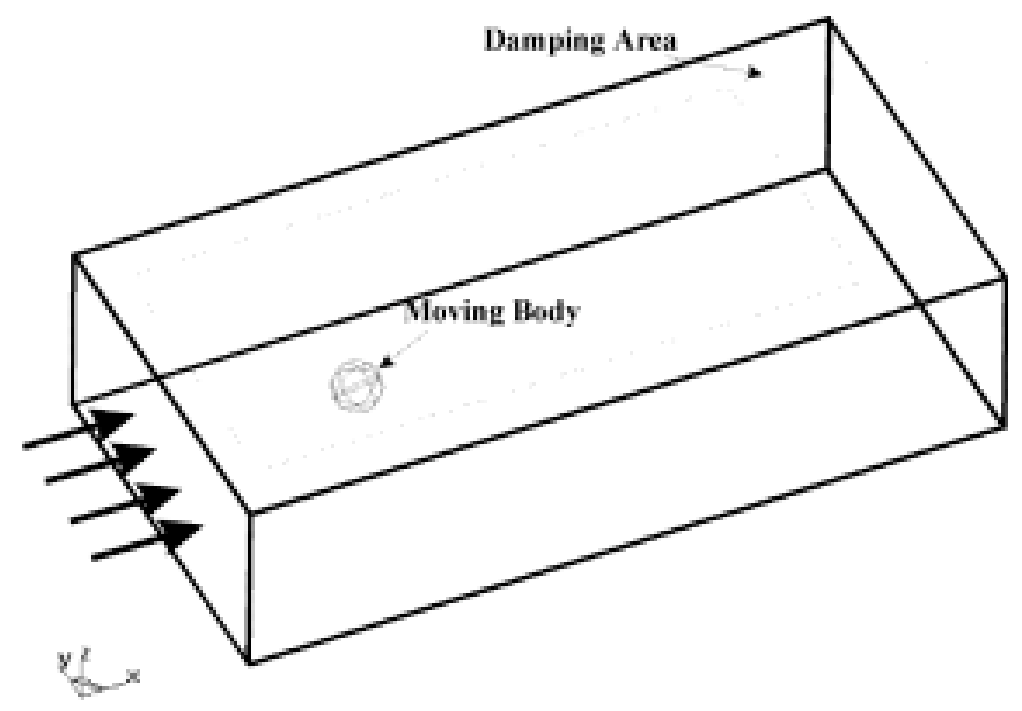

Figure 9. Geometry of the chanel with submerged sphere 


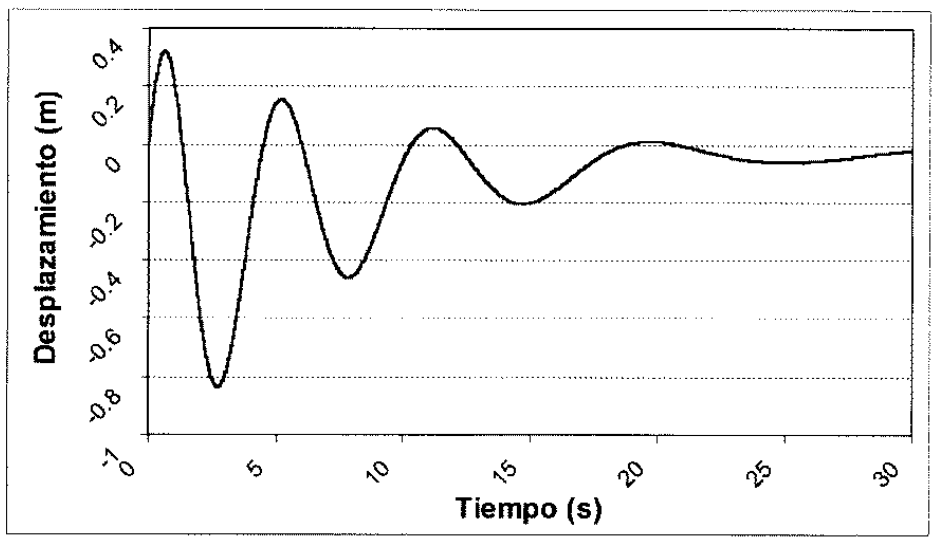

Figure 10. Time evolution of vertical displacement of sphere
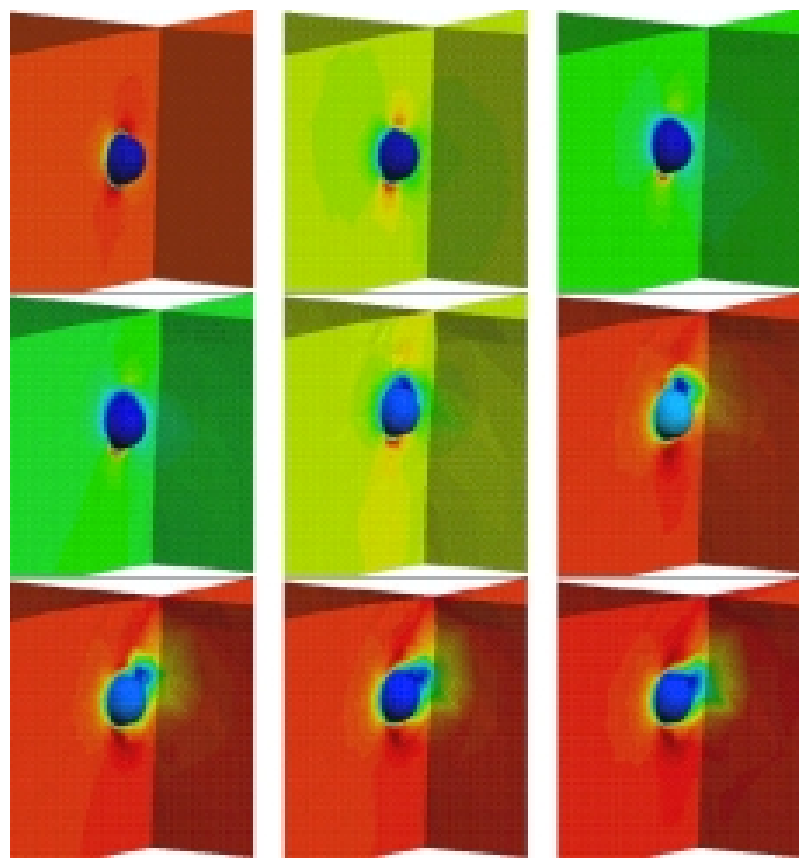

Figure 11. Contours of velocity module in the fluid on two perpendicular planes at different times 
Figure 12. Deformation of the free surface amplified 10 times at times $t=0.47 \mathrm{~s}$. and $t=3.16 \mathrm{~s}$.

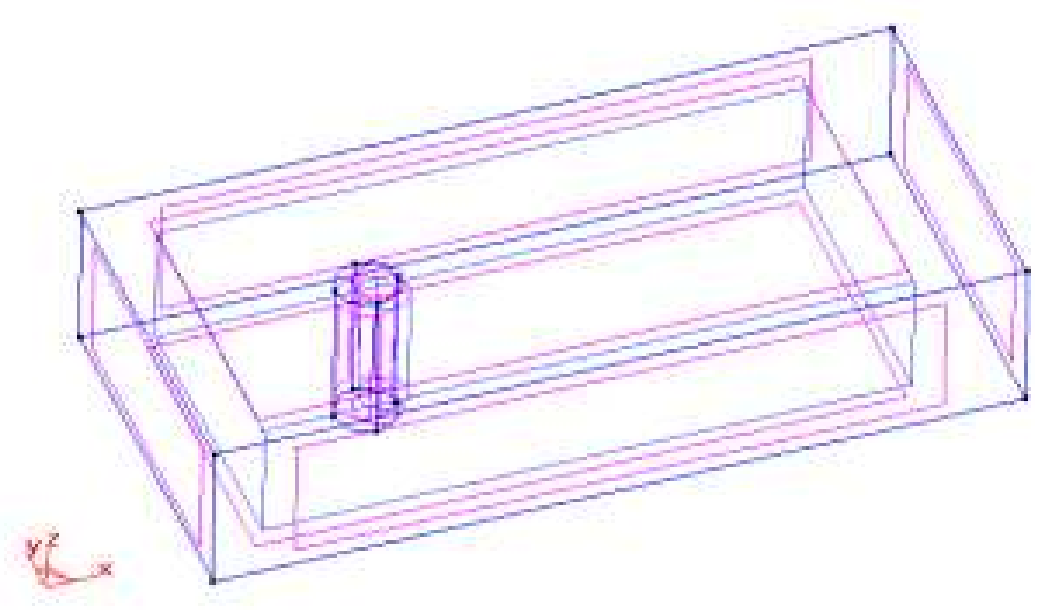

Figure 13. CAD definition of the vertical cylinder problem 


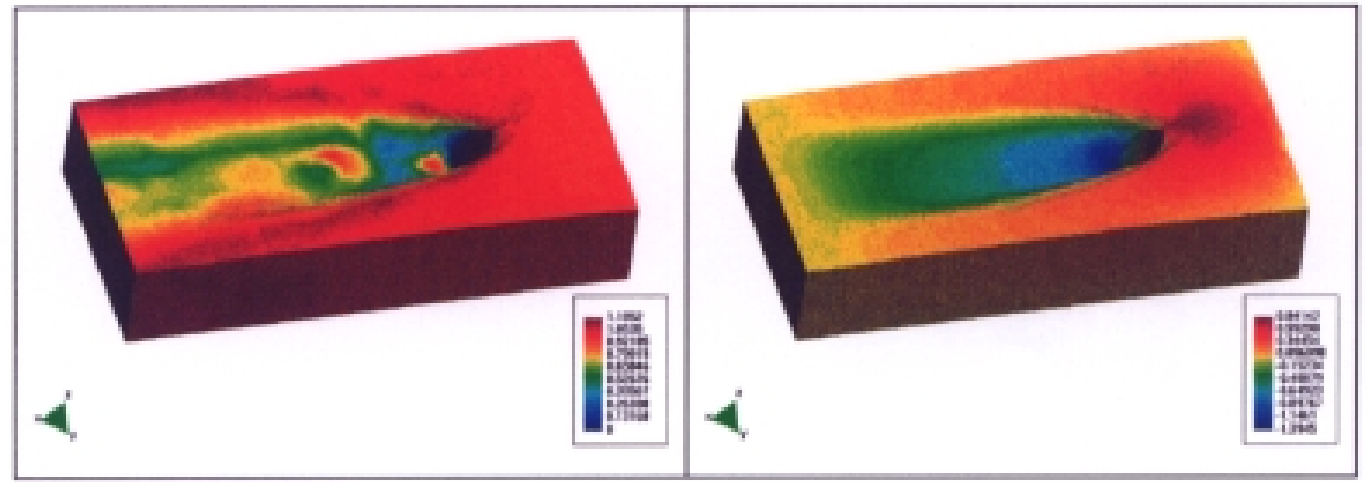

Figure 14. Vertical cylinder. Contours of velocity module and of vertical deformation of the mesh for $t=4.57 \mathrm{~s}$. 
Figure 15. Wigley hull. Pressure distribution, free surface contours and mesh deformation for the truly free ship motion 